\title{
Self-Assembled Asymmetric Microlenses for Four-Dimensional Visual Imaging
}

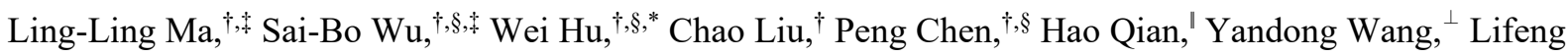

Chi, ${ }^{\perp}$ and Yan-qing $\mathrm{Lu}^{\dagger, *}$

${ }^{\dagger}$ National Laboratory of Solid State Microstructures, Key Laboratory of Intelligent Optical Sensing and Manipulation, College of Engineering and Applied Sciences, and Collaborative Innovation Center of Advanced Microstructures, Nanjing University, Nanjing 210093, China

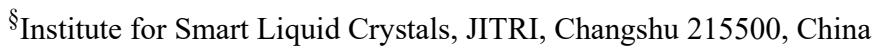

"State Key Laboratory of Materials Oriented Chemical Engineering, College of Materials Science and Engineering, Nanjing Tech University, Nanjing 210009, China

${ }^{\perp}$ Jiangsu Key Laboratory for Carbon-Based Functional Materials Institute of Functional Nano \& Soft Materials (FUNSOM), Soochow University, Suzhou 215123, China

"Corresponding Authors: E-mail: huwei@nju.edu.cn, E-mail: yqlu@nju.edu.cn.

*These authors contributed equally to this work. 


\section{Supplementary Text 1: Edge detection algorithm}

The edge direction is perpendicular to the direction of maximum intensity change (i.e., edge normal) and the edge strength is related to the local image contrast along the normal. Edge detection includes a variety of mathematical methods that aim at identifying points in a digital image at which the image brightness changes sharply or, more formally, has discontinuities. ${ }^{1}$ Here, we use the sum of edge strengths to measure the image definition by an edge detection algorithm based on the second-order derivatives of the grayscale. The steps to calculate the image definition are as follows:

1) Extract $28 \times 28$ pixels from the center of image and convert the RGB image to grayscale;

2) Apply differentiation to enhance the quality of edges (i.e., sharpening);

3) Calculate the sum of absolute values of edge strengths as the image definition

To simplify the algorithm, we chose the vertical bisectors of "E" and "O" images as the detection directions and use one-dimensional function of the second derivative:

$$
\begin{aligned}
& f^{\prime \prime}(x)=\lim _{h \rightarrow 0} \frac{f^{\prime}(x+h)-f^{\prime}(x)}{h} \approx f^{\prime}(x+1)-f^{\prime}(x) \\
& =f(x+2)-2 f(x+1)+f(x)(h=1) .
\end{aligned}
$$

Herein, $(x)$ denotes the $x$ position of the target pixel. $f(x)$ represents the grayscale value of this pixel. By replacing $x+1$ with $x$ (i.e., centered at $x$ ):

$$
f^{\prime \prime}(x) \approx f(x+1)-2 f(x)+f(x-1) .
$$

Thus the mask is $\left[\begin{array}{lll}1 & -2 & 1\end{array}\right]^{\mathrm{T}}$. On the basis of the second derivative, we optimize the above mask by extending its rows: $\left[\begin{array}{ll}1 & 1\end{array}\right.$ $\left.\begin{array}{llllll}1 & -8 & 1 & 1 & 1 & 1\end{array}\right]^{\mathrm{T}}$. Then for each pixel, the edge intensity is:

$$
\begin{aligned}
g(x)= & f(x-4)+f(x-3)+f(x-2)+f(x-1)+f(x+1) \\
& +f(x+2)+f(x+3)+f(x+4)-8 f(x) .
\end{aligned}
$$

The sum of absolute values of edge strengths (i.e., image definition, Def) can be written as:

$$
D e f=\sum|g(x)|
$$

\section{Supplementary Text 2: Optimization of the edge detection algorithm}

As shown in Figure $3 \mathrm{~h}$ and Figure S5h, other four regions with high imaging definition are observed at the four corners of the map. They are attributed to the partial lensing effect of nearly half the d-TFCD, exhibiting a shorter $f$ and an image position offset (Figure S6c). Therefore, the resultant images are deviated from the singularities of dTFCDs (Figure S5h and Figure S6b).

Considering the imaging ghosting and image position offset, we further optimize the edge detection algorithm via changing the detection-target area (i.e., $28 \times 28$ pixels) from the center of the " $\mathrm{E}$ " "OO" images to the region corresponding to the preset central area of the d-TFCD (Figure S6b). The imaging definitions after optimization are presented in Figure S6d.

\section{Supplementary Text 3: Triple-polarization imaging}

Triple-polarization imaging is also demonstrated by the multi- size and orientation d-TFCDs (Figure S7). Although the whole imaging quality is degraded by the mutual interference among patterns with different polarization components (Figure S7c), different polarization information is extracted successively by introducing an analyzer (Figure S7d-f). Details are presented in Supplementary Video. 


\section{Supplementary Figures}

a

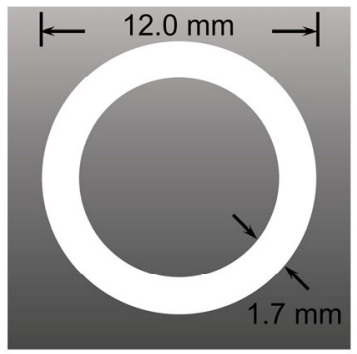

C

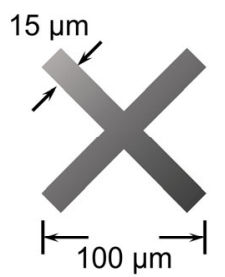

d

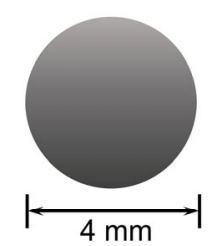

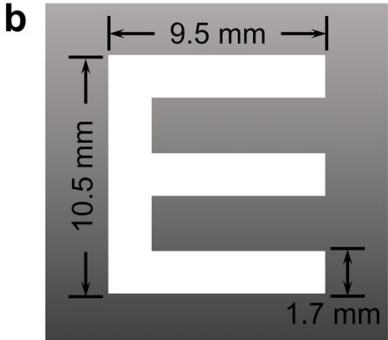

$\mathbf{e}$

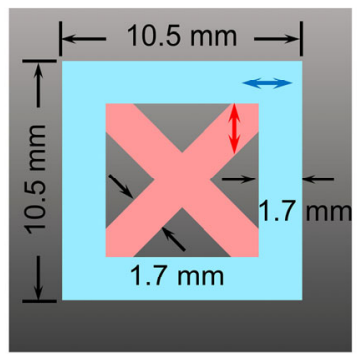

Figure S1. Objects for imaging. (a) A transmissive "O" mask with inner and external diameters of $8.6 \mathrm{~mm}$ and $12 \mathrm{~mm}$ respectively. (b) A transmissive "E" mask, $9.5 \mathrm{~mm} \times 10.5 \mathrm{~mm}$ in size and $1.7 \mathrm{~mm}$ in width. (c) An opaque cross, $100 \mu \mathrm{m} \times 100 \mu \mathrm{m}$ in size and $15 \mu \mathrm{m}$ in width. (d) An opaque disc with a diameter of $4 \mathrm{~mm}$. (e) Dual-polarization object. The cross is $7.1 \mathrm{~mm} \times 7.1 \mathrm{~mm}$ in size and $1.7 \mathrm{~mm}$ in width. The square is $10.5 \mathrm{~mm} \times 10.5 \mathrm{~mm}$ in size and $1.7 \mathrm{~mm}$ in width. Two orthogonal arrows indicate the transmitted polarization. 

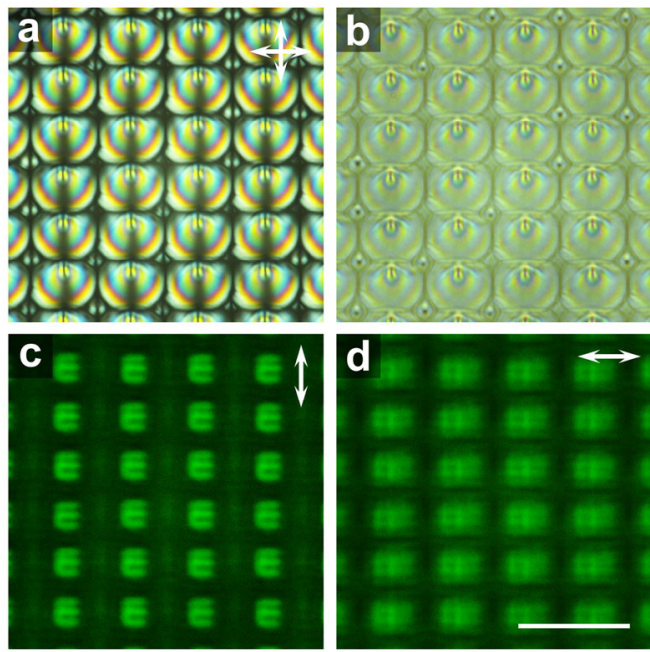

Figure S2. Polarization selectivity of d-TFCDs. (a) POM texture of d-TFCDs. $D=12.0 \mu \mathrm{m}$. The arrows indicate the optical axes of the crossed polarizers. (b) bright-field images of d-TFCDs. (c,d) Polarization-dependent imaging of d-TFCDs. $d_{o}=11.0 \mathrm{~cm}$ and $d_{i}=25.0$ $\mu \mathrm{m}$. Two arrows in the upper-right corners represent the polarization. The scale bar is $20 \mu \mathrm{m}$. 

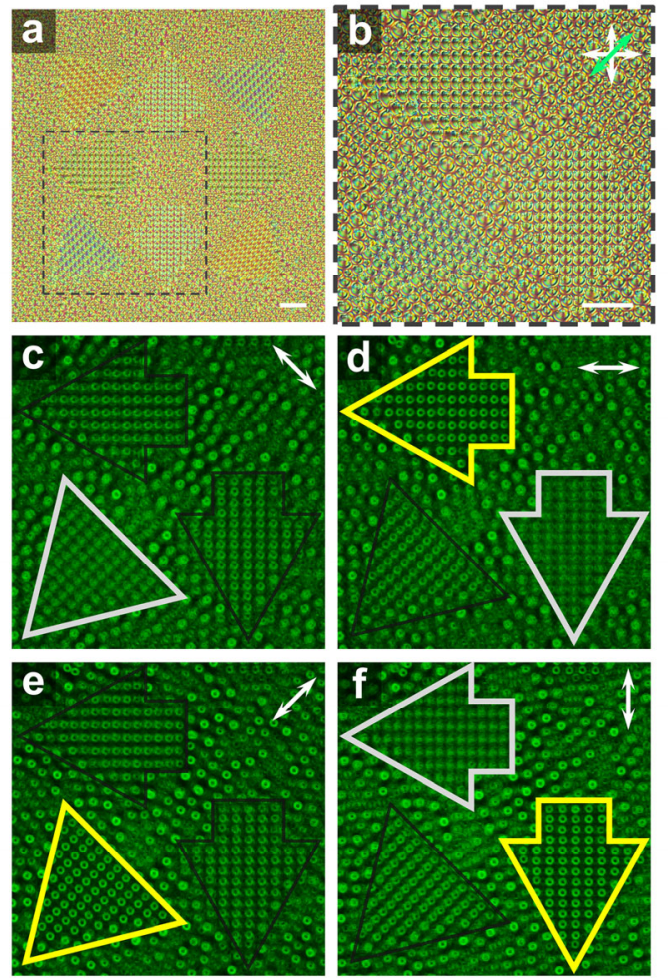

Figure S3. Details of the polarization selectivity of d-TFCDs. (a) POM texture of multidirectional d-TFCDs. The orientations of dTFCDs are consistent with arrows. The diameters of d-TFCDs are $12.0 \mu \mathrm{m}$. (b) Magnified POM texture in (a). (c-f) Magnified imaging pattern of a transmissive " $\mathrm{O}$ " mask for incident polarization of $135^{\circ}, 0^{\circ}, 45^{\circ}$ and $90^{\circ}$, separately. $d_{o}=11.0 \mathrm{~cm}$ and $d_{i}=25.0 \mu \mathrm{m}$. Regions with the clearest and fuzziest images are highlighted in yellow and grey respectively. All scale bars are $50 \mu \mathrm{m}$. 


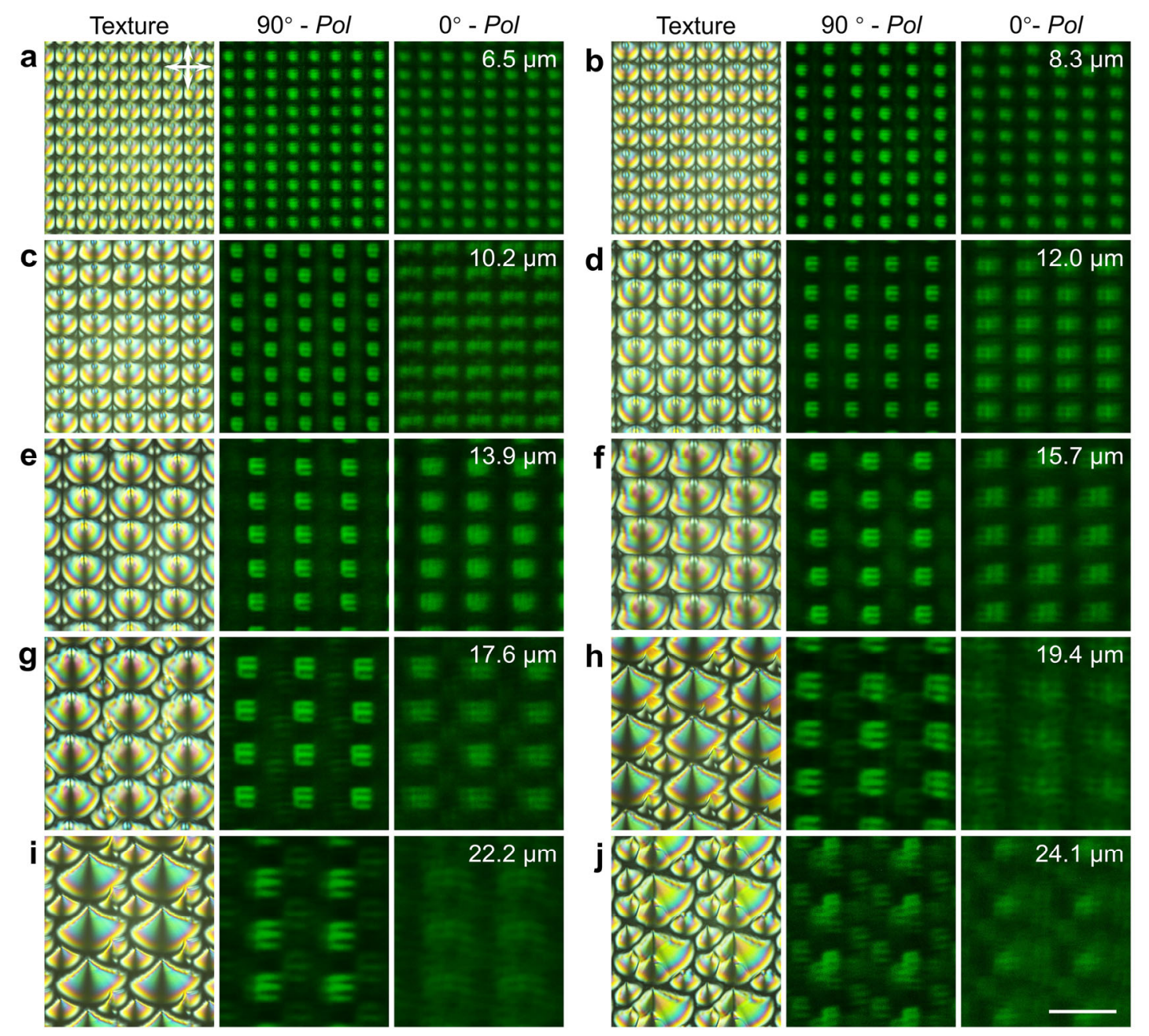

Figure S4. Multifocal functionality of d-TFCDs. POM textures and images of d-TFCDs. The diameters of d-TFCDs are labelled in the upper-right corners, (a) $6.5 \mu \mathrm{m}$, (b) $8.3 \mu \mathrm{m}$, (c) $10.2 \mu \mathrm{m}$, (d) $12.0 \mu \mathrm{m}$, (e) $13.9 \mu \mathrm{m}$, (f) $15.7 \mu \mathrm{m}$, (g) $17.6 \mu \mathrm{m}$, (h) $19.4 \mu \mathrm{m}$, (i) $22.2 \mu \mathrm{m}$ and (j) $24.1 \mu \mathrm{m}$. Pol denotes the incident polarization direction. The scale bar is $20 \mu \mathrm{m}$. 
a

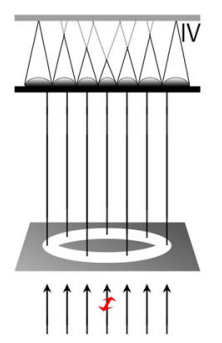

e

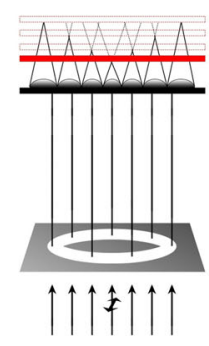

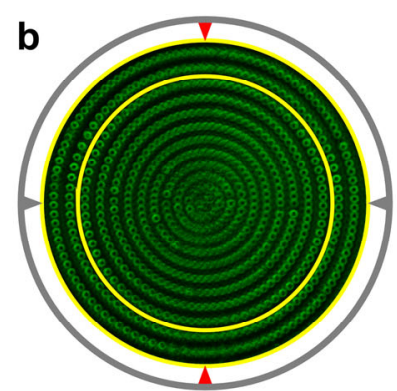

$\mathbf{f}$

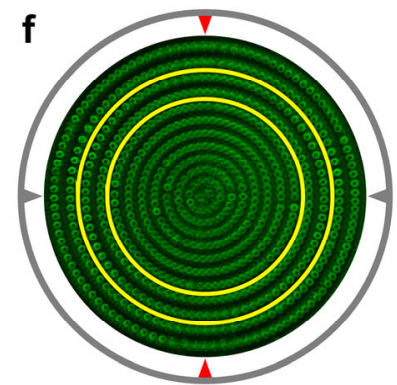

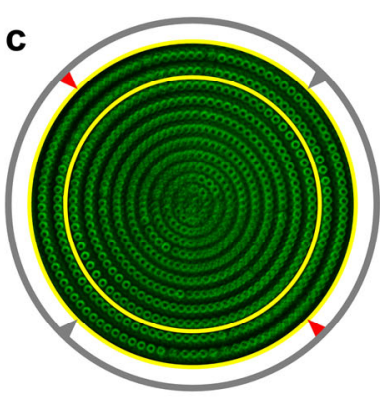

$\mathbf{g}$

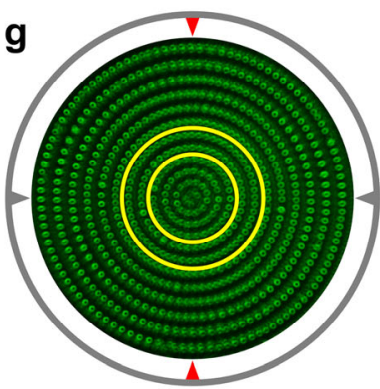

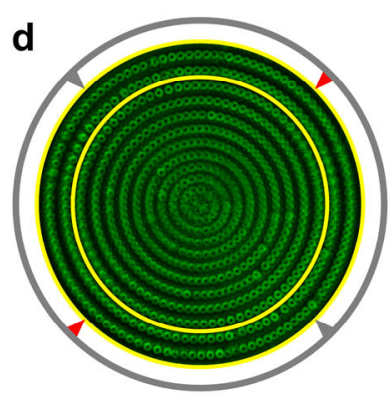

h

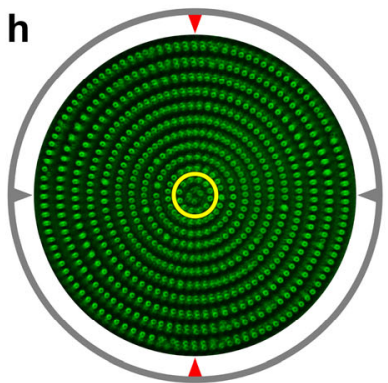

Figure S5. Extracting 4D information with multi- size and orientation d-TFCDs. (a) Schematic illustration of polarization-selective imaging with variable polarization. (b-d) Imaging patterns of the transmissive "O" mask. $\mathrm{Pol}$ are $90^{\circ}, 135^{\circ}$ and $45^{\circ}$, respectively. $d_{o}=$ $11.0 \mathrm{~cm}$ and $d_{i}=26.8 \mu \mathrm{m}$. (e) Schematic illustration of multifocal imaging with movable image plane. (f-h) Imaging patterns of the transmissive "O" mask. $d_{i}$ are $25.8 \mu \mathrm{m}, 23.0 \mu \mathrm{m}$ and $20.3 \mu \mathrm{m}$, respectively. $d_{o}=11.0 \mathrm{~cm}$ and $P o l=90^{\circ}$. "O" images on the most opportune image planes are circled in yellow. The grey and red pointers indicate the polar angles of the clearest images and corresponding incident beam polarization, respectively. 

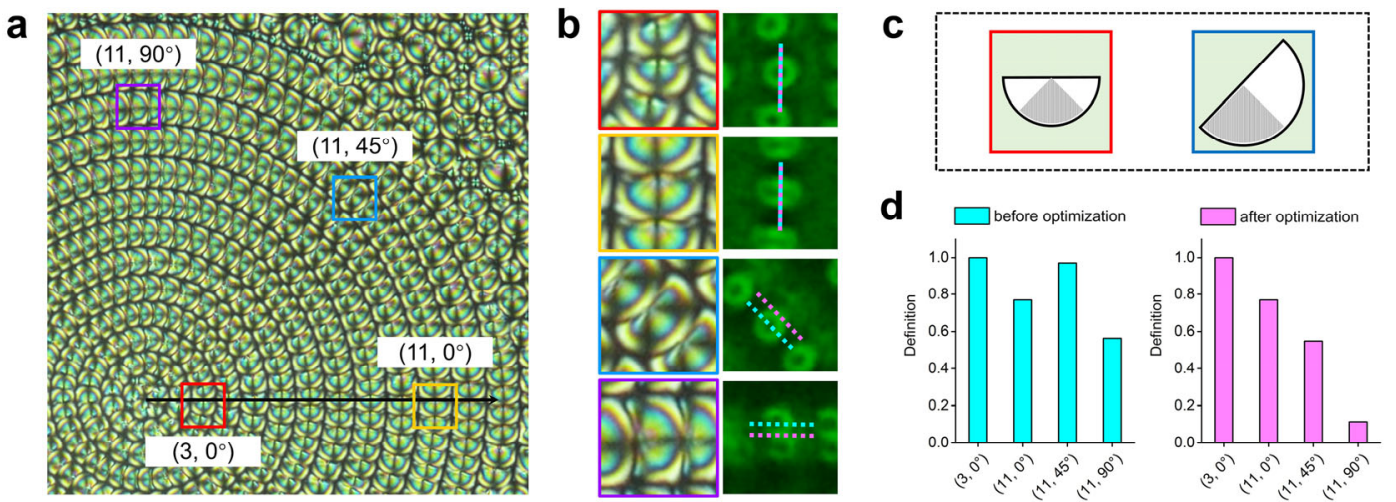

Figure S6. Partial lensing effect and optimization of imaging definition. (a) POM texture of the multi- size and orientation d-TFCDs. Colored squares indicate TFCDs with different coordinates $(r, \theta)$ labelled. (b) POM texture of local d-TFCDs and their "O" images. $d_{i}$ $=20.2 \mu \mathrm{m}$ and $\mathrm{Pol}=90^{\circ}$. Cyan and magenta dot lines denote the detection directions of the edge detection algorithm before and after optimization. (c) Schematic illustration of d-TFCDs at $\left(3,0^{\circ}\right)$ and $\left(11,45^{\circ}\right)$ for $90^{\circ}$ polarization. Shadow areas represent the effective parts in lensing effect. (d) Imaging definitions at different coordinates before or after optimization. 
a
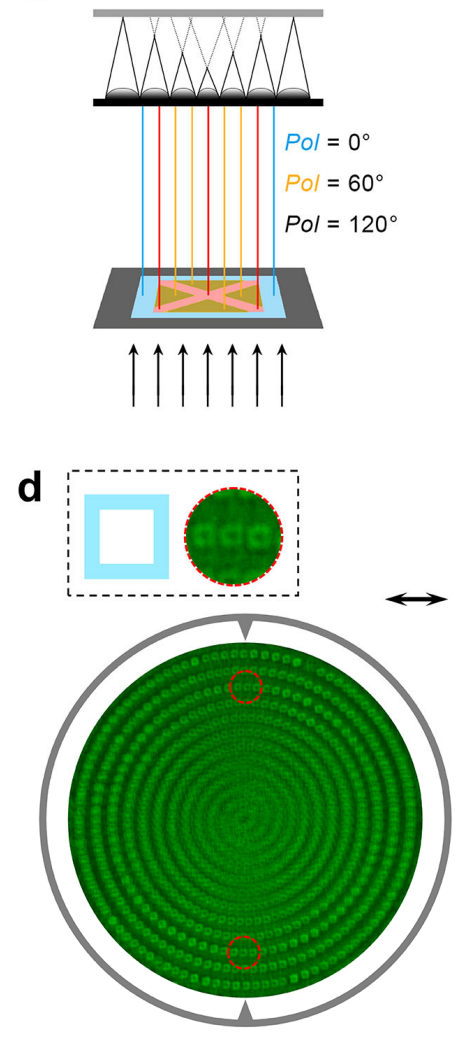

b

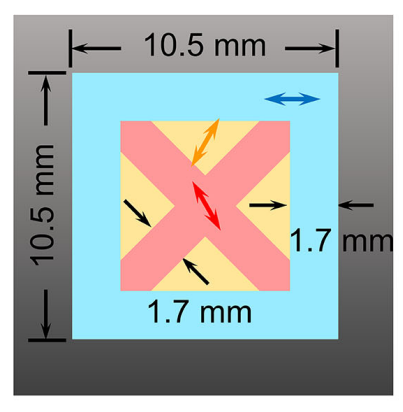

e
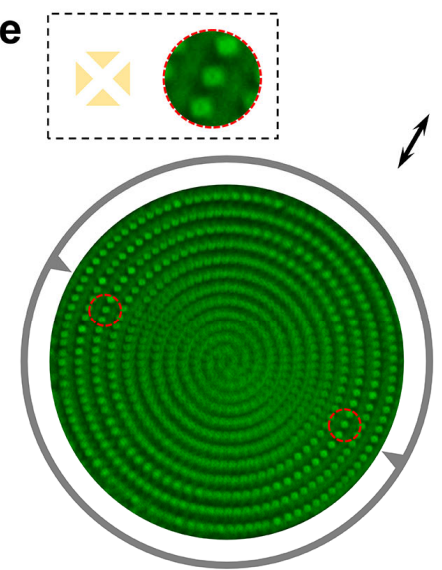

C

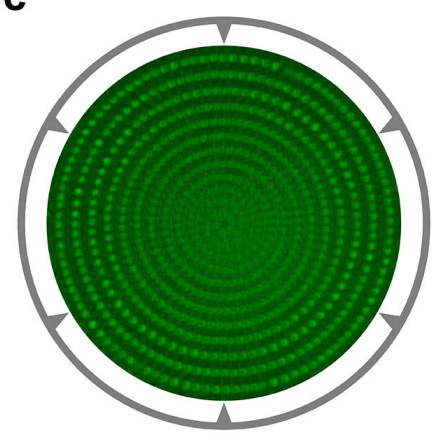

f

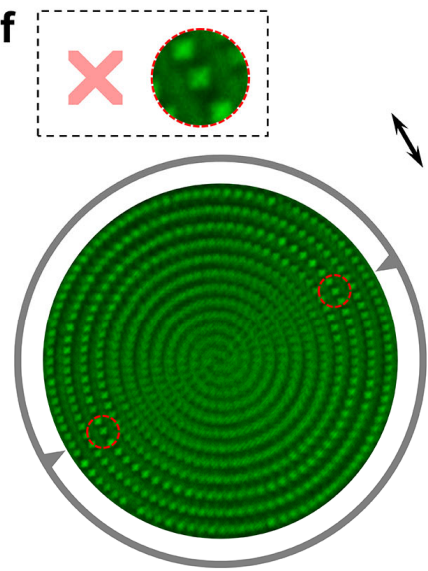

Figure S7. Triple-polarization imaging and polarization demultiplexing. (a) Schematic illustration of triple-polarization imaging. The polarization of square, triangles and cross are $0^{\circ}, 60^{\circ}$ and $120^{\circ}$, respectively. $d_{o}=11 \mathrm{~cm}$ and $d_{i}=25.7 \mu \mathrm{m}$. (b) Triple-polarization object. The square is $10.5 \mathrm{~mm} \times 10.5 \mathrm{~mm}$ in size and $1.7 \mathrm{~mm}$ in width. The cross is $7.1 \mathrm{~mm} \times 7.1 \mathrm{~mm}$ and $1.7 \mathrm{~mm}$ in width. Four triangles are complementary with the cross and square. (c) Imaging pattern of the object. (d-f) Extracted images with different polarization information through a rotatable analyzer above the object. Red circles label the clearest images, which are $200 \%$ amplified in the upsides. The grey pointers indicate the polar angles of the clearest images. The black arrows in the upper-right corners indicate the analyzer polarizations. 


\section{Supplementary Video}

Although the quality of triple-polarization imaging is degraded due to the mutual interference among different polarization components, each pattern with certain polarization information can be extracted successively by introducing an analyzer. As the analyzer is rotated counterclockwise, three different images emerge sequentially and their respective coordinates of the clearest images also varies counterclockwise. For instance, when the analyzer is rotated at $0^{\circ}$ (Figure S7d), the coordinates of the clearest square images are at $\left(10,90^{\circ} / 270^{\circ}\right)$. As shown in Figure S7e and $\mathrm{f}$, the coordinates of the clearest triangle images are at $\left(10,150^{\circ} / 330^{\circ}\right)$, and the coordinates of the clearest cross images are at $\left(10,210^{\circ} / 30^{\circ}\right)$. Corresponding polarization directions of the analyzer are $60^{\circ}$ and $120^{\circ}$, respectively. The rotational arrow in the video indicates the direction of the analyzer. The clearest images are highlighted with white square at $7 \mathrm{~s}, 15 \mathrm{~s}$ and $22 \mathrm{~s}$, which are $200 \%$ amplified in the lower-right corner.

\section{Supplementary Reference}

1. Gonzalez R. C.; Woods R. E. Digital Image Processing; Prentice Hall: New Jersey, 2002. 